\title{
Curcumin induces apoptosis and protective autophagy in castration-resistant prostate cancer cells through iron chelation
}

This article was published in the following Dove Press journal:

Drug Design, Development and Therapy

14 February 2017

Number of times this article has been viewed

\author{
Chunguang Yang ${ }^{1, *}$ \\ Xueyou Ma',* \\ Zhihua Wang' \\ Xing Zeng' \\ Zhiquan $\mathrm{Hu}^{\prime}$ \\ Zhangqun $\mathrm{Ye}^{\prime}$ \\ Guanxin Shen ${ }^{2}$ \\ 'Department of Urology, Tongji \\ Hospital, ${ }^{2}$ Department of Immunology, \\ Tongji Medical College, Huazhong \\ University of Science and Technology, \\ Wuhan, Hubei, People's Republic \\ of China \\ *These authors contributed equally \\ to this work
}

\begin{abstract}
Background: Curcumin induces apoptosis and autophagy in different cancer cells. Moreover, chemical and biological experiments have evidenced that curcumin is a biologically active iron chelator and induces cytotoxicity through iron chelation. We thus hypothesized that curcumin may induce apoptosis and autophagy in castration-resistant prostate cancer (CRPC) cells through its iron-chelating properties.
\end{abstract}

Materials and methods: CRPC cells were loaded with curcumin alone or in combination with ferric ammonium citrate (FAC). Cytotoxicity was measured by 3-(4,5-dimethylthiazol2-yl)-2,5-diphenyltetrazolium bromide (MTT) assay. Apoptosis was assessed by flow cytometry, terminal deoxynucleotidyl transferase nick end labeling (TUNEL) assay and caspase activity. Autophagy status was analyzed by the detection of autophagosomes and light chain 3-II (LC3-II) using transmission electron microscopy and Western blot. Iron-binding activity of curcumin was assessed by spectrophotometry and MTT assay. The expression levels of transferrin receptor 1 (TfR1) and iron regulatory protein 1 (IRP1) were examined by Western blot.

Results: Curcumin induced apoptosis and autophagy in CRPC cells. Combining curcumin with autophagy inhibitors (3-methyladenine [3-MA]) synergized the apoptotic effect of curcumin. Moreover, curcumin bound to FAC at a ratio of $\sim 1: 1$, as assessed by spectrophotometry and MTT assay. Apoptosis and autophagy induced by curcumin were counteracted by equal amounts of FAC. At apoptosis- and autophagy-inducing concentrations, curcumin enhanced the expression levels of TfR1 and IRP1, indicative of iron deprivation induced by curcumin.

Conclusion: Together, our results indicate that curcumin induces apoptosis and protective autophagy in CRPC cells, which are at least partially dependent on its iron-chelating properties.

Keywords: curcumin, castration-resistant prostate cancer, apoptosis, autophagy, iron chelation

\section{Introduction}

Prostate cancer (PCa) is the second most frequently diagnosed cancer and the fifth leading cause of cancer-related death among men worldwide. ${ }^{1}$ Advanced PCa responds to androgen deprivation therapy (ADT) for an average duration of 14-20 months but then may eventually proceed to hormone-refractory disease, termed castrationresistant prostate cancer (CRPC). ${ }^{2}$ Better and more effective therapies against CRPC are urgently needed.

Iron is an essential component of many enzymes that play important biological roles in all cellular forms of life. ${ }^{3}$ Intracellular iron levels are tightly regulated to balance between iron acquisition, storage and usage. ${ }^{4}$ Mammalian cells mainly acquire iron from plasma transferrin (Tf). Then, iron-loaded Tf binds with transferrin
Correspondence: Zhihua Wang Department of Urology, Tongji Hospital, Tongji Medical College, Huazhong University of Science and Technology, 1095 Jiefang Avenue, Wuhan 430030, People's Republic of China

Tel +862783665308 Fax +862783665368

Email zhwang_hust@hotmail.com (c) (1) (5) 2017 Yang et al. This work is published and licensed by Dove Medical Press Limited. The full terms of this license are available at https://www.dovepress.com/terms.php (c) $\mathrm{BY}$ and incorporate the Creative Commons Attribution - Non Commercial (unported, v3.0) License (http:///creativecommons.org/licenses/by-nd/3.0/). By accessing the work you hereby accept the Terms. Non-commercial uses of the work are permitted without any further permission from Dove Medical Press Limited, provided the work is properly attributed. For permission for commercial use of this work, please see paragraphs 4.2 and 5 of our Terms (https://www.dovepress.com/terms.php). 
receptor 1 (TfR1) on the cell surface, undergoes endocytosis and releases iron from Tf to the cytoplasm, where ferritin is synthesized for iron storage. Under iron deficiency conditions, the iron regulatory proteins (IRPs) bind to the iron response elements (IREs) of ferritin and TfR 1 mRNAs. The IRE-IRP interactions inhibit the translation of ferritin mRNA but stabilize TfR1 mRNA, thereby inhibiting ferritin synthesis and enhancing the expression of TfR1. Thus, the expression levels of TfR1, ferritin and iron regulatory protein 1 (IRP1) can indirectly reflect the intracellular status of iron. Epidemiological studies and animal experiments have reported an association between iron overload and carcinogenesis. ${ }^{5}$ Moreover, as iron deprivation has shown to induce apoptosis and autophagy in a series of cell lines, this may implicate iron deprivation as a promising strategy for cancer therapy investigation. ${ }^{6,7}$

Derived from the roots of Curcuma longa, curcumin possesses anticancer, anti-inflammatory, antioxidative and antimicrobial properties. ${ }^{8}$ Studies have shown that curcumin induces caspase-related apoptosis in both androgendependent and castration-refractory $\mathrm{PCa}$ cells, as opposed to autophagy alone in androgen-dependent PCa cells..$^{9,10}$ Moreover, chemical and biological experiments have shown that curcumin is a biologically active iron chelator and induces cytotoxicity through iron chelation. ${ }^{11,12}$ These findings indicate that curcumin may induce apoptosis and autophagy in CRPC cells through its iron-chelating properties.

In the present study, we investigated whether curcumin induces autophagy in CRPC cells, and if so, whether ironchelating properties of curcumin are part of the mechanism by which it induces apoptosis and autophagy.

\section{Materials and methods Cell culture and chemicals}

The CRPC cell lines (DU145 and PC3) were obtained from the American Type Culture Collection (ATCC, Manassas, VA, USA). Cells were cultured in Dulbecco's Modified Eagle's Medium (DMEM; Thermo Fisher Scientific, Waltham, MA, USA) supplemented with $10 \%$ fetal bovine serum (FBS; Gibco) at $37^{\circ} \mathrm{C}$ in a $5 \% \mathrm{CO}_{2}$ humidified atmosphere. Curcumin ((E,E)-1,7bis(4-hydroxy-3-methoxyphenyl)-1,6-heptadiene-3, 5-dione, CAS number: 458-37-7; Sigma-Aldrich, St Louis, MO, USA) was dissolved in dimethyl sulfoxide (DMSO) (Sigma-Aldrich). 3-Methyladenine (3-MA; Selleck Chemicals, Houston, TX, USA) was dissolved in Eagle's Minimal Essential Medium (EMEM; Seebio Biotech,
Shanghai, China). Antibodies were obtained as follows: light chain 3-II (LC3-II; Proteintech Group, Rosemont, IL, USA), caspase-3 (Proteintech Group), caspase-9 (Santa Cruz, Dallas, TX, USA), transferring receptor monoclonal antibody (TfR1) (a kind gift from Professor Guanxin Shen, purified as described ${ }^{13}$ ), IRP1 (Proteintech Group), $\beta$-actin (Santa Cruz) and horseradish peroxidase (HRP)conjugated goat anti-rabbit secondary antibody (Santa Cruz).

\section{Cell viability}

Cell viability was assessed by 3-(4,5-dimethylthiazol2-yl)-2,5-diphenyltetrazolium bromide (MTT) assay (Sigma-Aldrich). ${ }^{14}$ DU145 and PC3 cells were cultured in 96-well plates and incubated with different concentrations of curcumin alone or in combination with ferric ammonium citrate (FAC) (Sigma-Aldrich) at different ratios for $24 \mathrm{~h}$ or pretreated with $2 \mathrm{mM} 3$-MA for $1 \mathrm{~h}$. Absorbance measurements of each well were taken at $570 \mathrm{~nm}$. All experiments were performed in triplicate. The cell viability was expressed as a percentage of the control.

\section{Apoptosis assay}

Annexin V-affinity assay is an apoptosis detection system based on phosphatidylserine (PS) exposure. DU145 and PC3 cells were plated into 12-well plates and treated with curcumin $(50 \mu \mathrm{M})$ alone or in combination with FAC $(50 \mu \mathrm{M})$ or pretreated with 3-MA $(2 \mathrm{mM}, 1 \mathrm{~h})$ for $24 \mathrm{~h}$. Tumor cells were then washed with ice-cold phosphate-buffered saline (PBS) and detected by flow cytometry (FCM; BD Biosciences, San Jose, CA, USA), following the protocols of Annexin V-fluorescein isothiocyanate Apoptosis Detection Kit (Roche Diagnostics, Mannheim, Germany). ${ }^{15}$

\section{Fluorescence microscopy}

DU145 and PC3 cells were treated with $50 \mu \mathrm{M}$ curcumin alone or in combination with $50 \mu \mathrm{M} \mathrm{FAC}$ for $24 \mathrm{~h}$. Apoptotic cells were determined by terminal deoxynucleotidyl transferase (TdT)-mediated deoxyuridine triphosphate (dUTP)biotin nick end labeling (TUNEL; Roche Diagnostics). Tumor cells were washed twice with ice-cold PBS, fixed with $4 \%$ paraformaldehyde for $40 \mathrm{~min}$ at room temperature and permeabilized in $0.1 \%$ Triton $\mathrm{X}-100$ in $0.1 \%$ sodium citrate for $2 \mathrm{~min}$. Specimens were then incubated with a mixture of TdT solution and FITC-deoxyuridine triphosphate $(\mathrm{dUTP})$ solution at $37^{\circ} \mathrm{C}$. The negative controls were incubated with distilled water in place of TdT enzyme. 
To visualize cell nuclei, the samples were stained with 4 , 6-diamidino-2-phenylindole dihydrochloride (DAPI; Roche Diagnostics) and examined microscopically at $400 \times$ magnification. All experiments were conducted in triplicate.

\section{Transmission electron microscopy (TEM)}

DU145 and PC3 cells were treated with $50 \mu \mathrm{M}$ curcumin for $24 \mathrm{~h}$. Then, tumor cells were washed twice with ice-cold PBS, fixed in $2.5 \%$ glutaraldehyde for $60 \mathrm{~min}$ at room temperature and post-fixed in 1\% osmium tetroxide for $30 \mathrm{~min}$. After being embedded in Epon 812 resin, ultrathin sections were stained with uranyl acetate and lead citrate. The ultrastructure was examined using a transmission electron microscope (HT7700; Hitachi, Tokyo, Japan) operated at $5 \mathrm{kV}$.

\section{Spectrophotometry}

Increasing concentrations of FAC were incubated in 96-well plates with or without $50 \mu \mathrm{M}$ curcumin for $30 \mathrm{~min}$, and the absorbance of each well at $430 \mathrm{~nm}$ was examined with the enzyme-linked immunosorbent assay (ELISA) reader. All experiments were performed in triplicate.

\section{Western blot}

Total proteins were extracted from the cell pellets by homogenization in Radio Immunoprecipitation Assay (RIPA) lysis buffer containing protease inhibitor cocktail (SigmaAldrich) for $30 \mathrm{~min}$ on ice. Concentrations were determined using the BCA Protein Assay Kit (Pierce, Rockford, IL, USA). Protein samples (20-60 $\mu \mathrm{g})$ were loaded on $4 \% / 10 \%$ sodium dodecyl sulfate polyacrylamide gel electrophoresis (SDS-PAGE) gels and subjected to electrophoretic analysis and subsequent blocking. Membranes were incubated with the primary antibody (overnight at $4^{\circ} \mathrm{C}$ ) and the relevant secondary antibodies ( $1 \mathrm{~h}$ at room temperature). Western blot was conducted to analyze the expression levels of caspase-3, caspase-9, LC3-II, TfR1 and IRP1 in tumor cells. The blots were exposed to autoradiography films (X-OMAT BT; Eastman Kodak, Rochester, NY, USA). The protein band density was quantified using the ImageJ software (Version 1.38x; National institutes of Health, Bethesda, MD, USA), with $\beta$-actin as a loading control. The relative expression of proteins was expressed as the ratio of their expression to $\beta$-actin expression. All experiments were conducted in triplicate.

\section{Statistical analysis}

Statistical analysis was performed using the SPSS 12.0 software (SPSS Inc., Chicago, IL, USA). Data are represented as mean $\pm \mathrm{SD}$. Student's $t$-test was used to determine the statistical significance of differences between the test groups and controls. A $P$-value $<0.05$ was considered a statistically significant difference.

\section{Results}

\section{Curcumin induces caspase-related apoptosis in CRPC cells}

To confirm the apoptosis-inducing effects of curcumin, we tested its impacts on PS exposure and caspase activation in CRPC cells. The cells were incubated in a growth medium with $0,6.25,12.5,25$ and $50 \mu \mathrm{M}$ curcumin for $24 \mathrm{~h}$. The results of FCM showed that curcumin induced a dose-dependent apoptosis in DU145 and PC3 cells (Figure 1A), accompanied by expression of caspase- 3 and caspase- 9 as shown by Western blot (Figure 1B). The results indicated that curcumin induces caspase-related apoptosis in CRPC cells, consistent with the current studies performed by Mathur et al. ${ }^{16}$ Moreover, both TUNEL-positive cells observed in curcumin-treated cultures and chromatin condensation detected by DAPI staining confirmed that curcumin induced apoptosis in DU145 and PC3 cells (Figure 2A and B, respectively).

\section{Curcumin induces autophagy in CRPC cells}

Then, we investigated the effect of curcumin on autophagy. After culturing in $50 \mu \mathrm{M}$ curcumin for $24 \mathrm{~h}$, characteristic multiple membrane structures (autophagosomes) were observed in CRPC cells by TEM (Figure 3A and B). These observations were corroborated by the evaluated expression of autophagy markers LC3-II in a dose-dependent manner (Figure 3C). These results suggest that curcumin activated autophagy in DU145 and PC3 cells.

\section{Inhibition of autophagy increased the cytotoxicity of curcumin}

The above results revealed that curcumin induced both apoptosis and autophagy in CRPC cells. We then evaluated whether there was a relationship between apoptosis and autophagy induced by curcumin. To elucidate the role of autophagy induced by curcumin, we investigated the effect of 3-MA on autophagy and apoptosis. 3-MA is a commonly used reagent that can inhibit autophagy by blocking autophagosome formation. Cytotoxicity of curcumin in DU145 and PC3 cells increased significantly when combined with $2 \mathrm{mM}$ 3-MA (Figure 4A and B, respectively). Moreover, the rate of apoptosis induced by curcumin increased distinctly with 


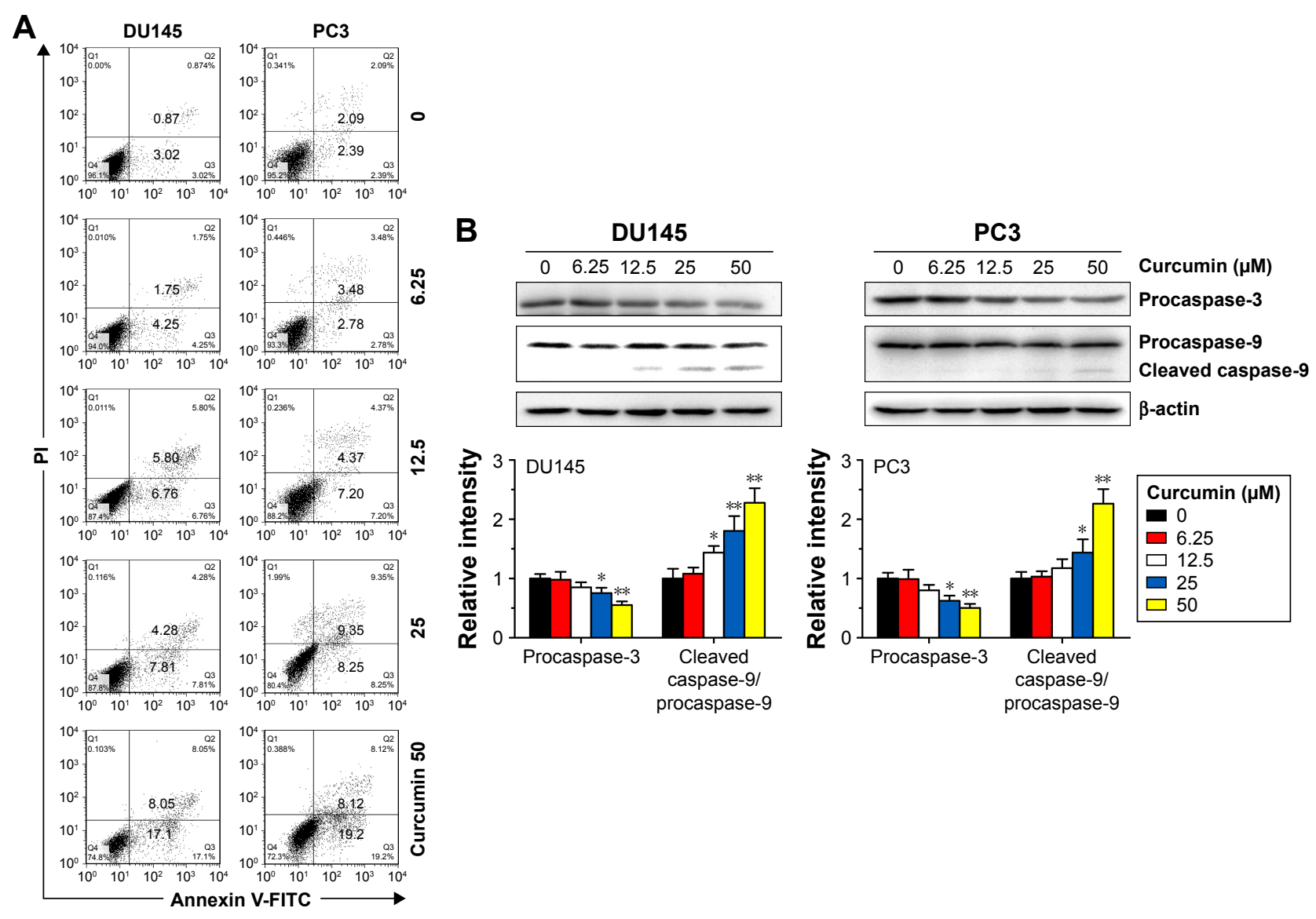

Figure I Curcumin induces caspase-related apoptosis in DUI 45 and PC3 cells.

Notes: (A) DUI 45 and PC3 cells were treated with various concentrations of curcumin (0-50 $\mu \mathrm{M})$ for $24 \mathrm{~h}$, and the percentage of apoptotic cells was analyzed by FCM analysis of Annexin V-FITC and PI double staining. (B) Expression levels of caspase-3 and caspase-9 were examined by Western blot analysis. $n=3, * P<0.05$ and $* * P<0.01$ vs control, by Student's $t$-test.

Abbreviations: FCM, flow cytometry; FITC, fluorescein isothiocyanate; PI, propidium iodide.

A
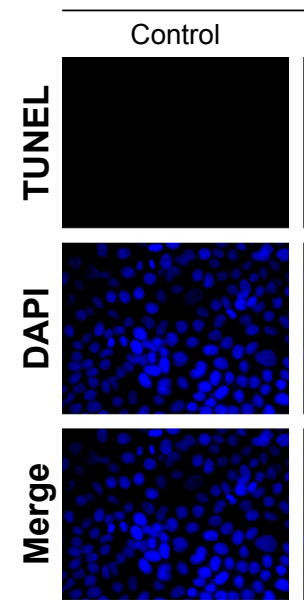

DU145
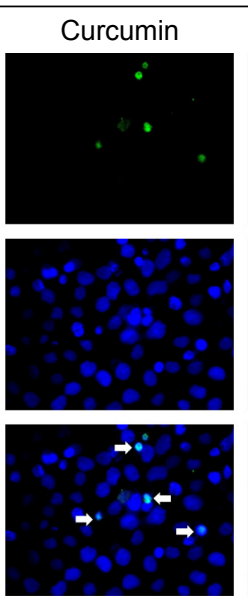
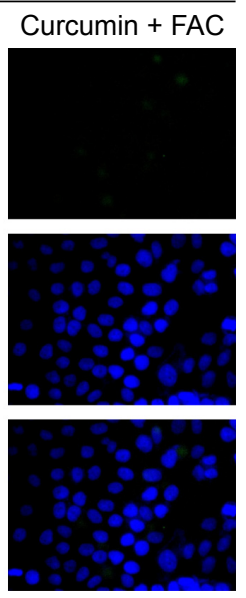

B
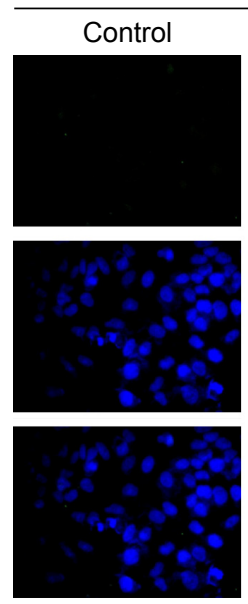

PC3
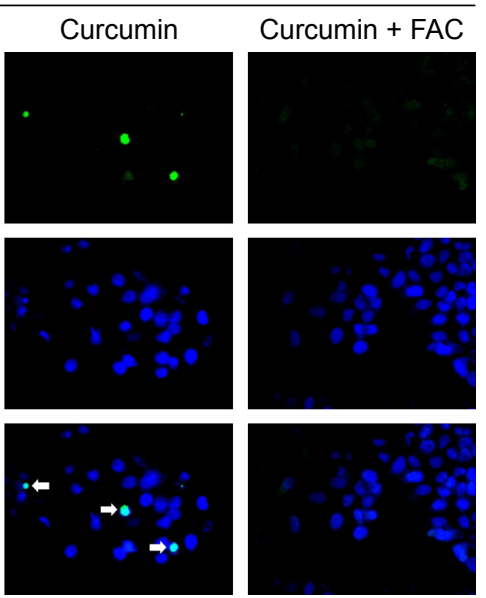
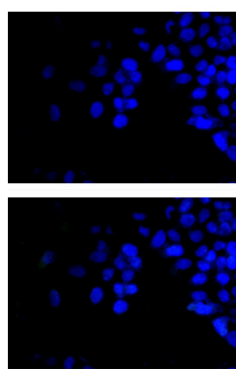

Figure 2 Morphological study with a fluorescence microscope.

Notes: Cultured DUI 45 (A) and PC3 (B) cells were incubated with curcumin (50 $\mu$ M) or combined with FAC (50 $\mu$ M) for 24 h and then stained with FITC-labeled TUNEL and DAPI. The magnification was $400 \times$.

Abbreviations: FAC, ferric ammonium citrate; FITC, fluorescein isothiocyanate; TUNEL, terminal deoxynucleotidyl transferase (TdT)-mediated deoxyuridine triphosphate (dUTP)-biotin nick end labeling; DAPI, 4,6-diamidino-2-phenylindole dihydrochloride. 
A

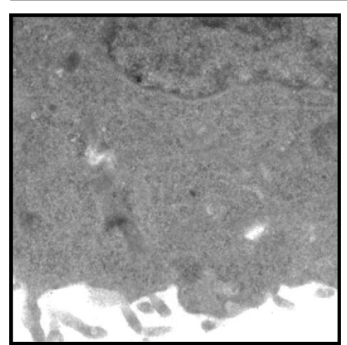

Control
DU145

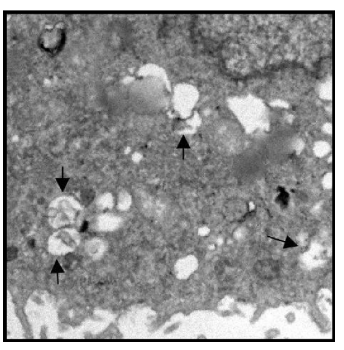

Curcumin
B

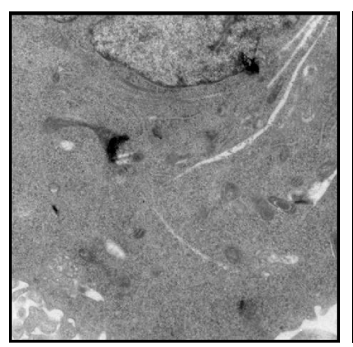

Control
PC3

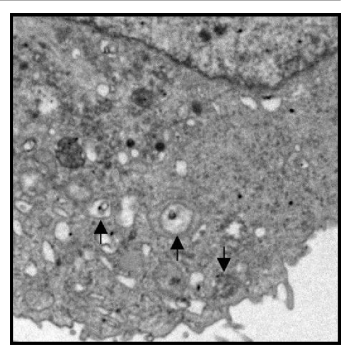

Curcumin

C

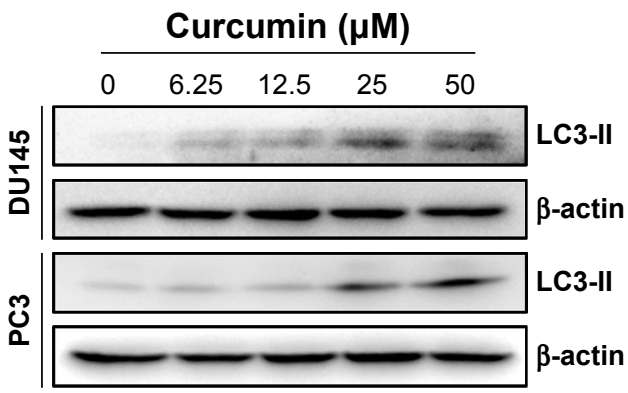

Figure 3 Curcumin induces autophagy in DUI45 and PC3 cells.

Notes: DUI45 (A) and PC3 (B) showed characteristic autophagosomes after incubation with curcumin (50 $\mu$ M) for 24 h, as highlighted by black arrows. (C) DUI45 and $P C 3$ cells were incubated with curcumin at different concentrations $(\mu \mathrm{M})$ for $24 \mathrm{~h}$, and expression of LC3-II was examined by Western blot analysis. $\mathrm{n}=3$, $* P<0.05$, $* * P<0.0$ I and $* * * P<0.001$ vs control, by Student's $t$-test.

Abbreviation: LC3-II, light chain 3-II.

A

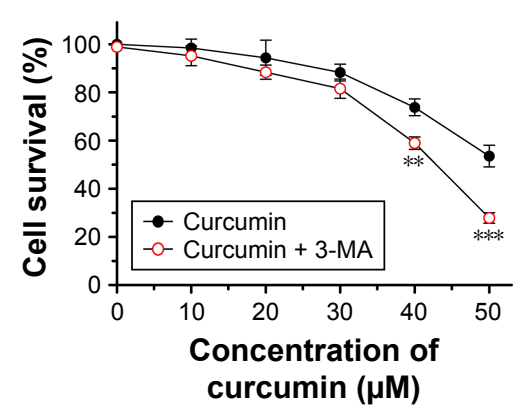

D

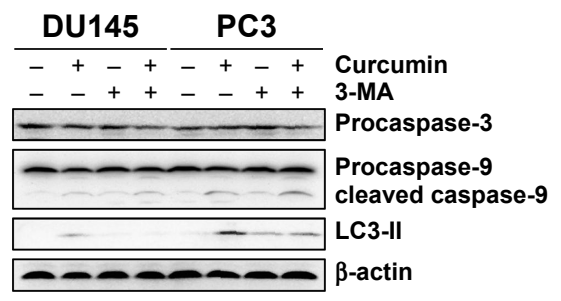

B

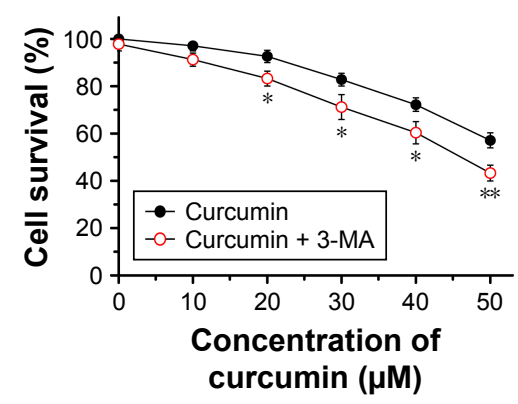

DU145

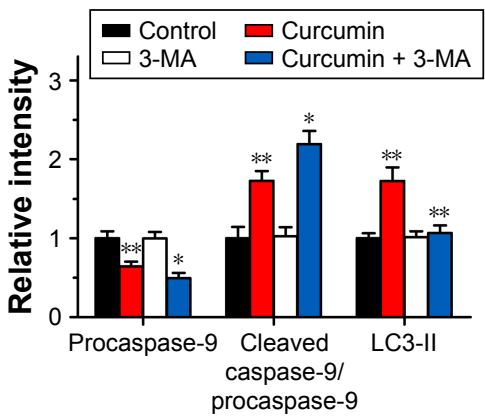

C

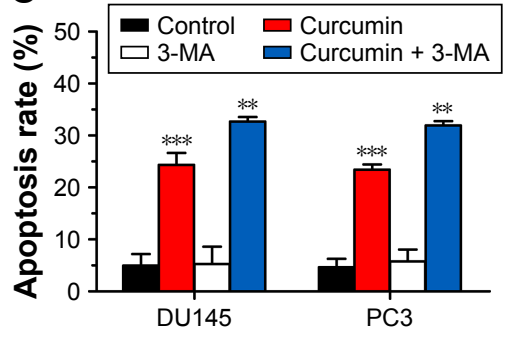

PC3

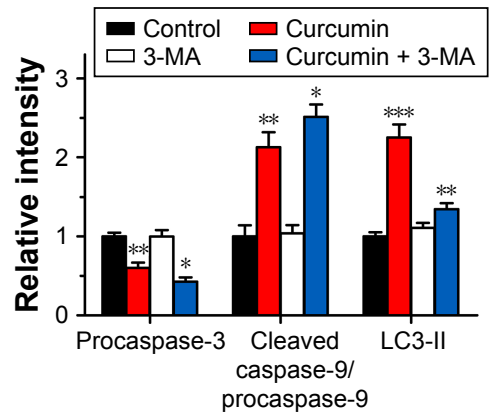

Figure 4 3-MA enhances the apoptosis-inducing activity of curcumin.

Notes: DUI45 (A) and PC3 (B) cells were incubated for $24 \mathrm{~h}$ with various concentrations of curcumin mixed with 3-MA at 2 mM. Survival rates of DUI45 and PC3 cells were determined by MTT assay. The data are expressed as mean \pm SD. $n=3, * P<0.05, * * P<0.0$ I and $* * * P<0.00$ I vs curcumin-treated group, by Student's $t$-test. (C) DUI 45 and PC3 cells were treated with curcumin $(50 \mu \mathrm{M})$ or combined with 3-MA ( $2 \mathrm{mM})$ for $24 \mathrm{~h}$, and the percentage of apoptotic cells was analyzed by FCM analysis of Annexin $\mathrm{V}$ and PI double staining. The data are expressed as mean \pm SD. $n=3, * * P<0.0 I$ and $* * * P<0.001$ vs control or curcumin-treated group, by Student's $t$-test. (D) Expression levels of caspase- 3 and caspase- 9 were examined by Western blot analysis. $n=3, * P<0.05, * * P<0.01$ and $* * * p<0.001$ vs control or curcumin-treated group, by Student's $t$-test. Abbreviations: 3-MA, 3-methyladenine; MTT, 3-(4,5-dimethylthiazol-2-yl)-2,5-diphenyltetrazolium bromide; FCM, flow cytometry; PI, propidium iodide; LC3-II, light chain 3-II. 
the existence of 3-MA, which was accompanied with further activation of caspase-3 and caspase-9 (Figure 4C and D). These results demonstrated that autophagy induced by curcumin could repress apoptosis induced by curcumin in CRPC cells. In other words, curcumin induced protective autophagy in CRPC cells.

\section{Curcumin binds to FAC at a ratio of $\sim 1: 1$}

Evidence from absorption spectra has shown that the absorption peak of curcumin at $430 \mathrm{~nm}$ was significantly higher than that of curcumin-Fe(III) complexes in neutral solution. ${ }^{11}$ To investigate the iron-chelating activity of curcumin, increasing concentrations of FAC were incubated in 96-well plates with or without $50 \mu \mathrm{M}$ curcumin for $30 \mathrm{~min}$. Absorbance at $430 \mathrm{~nm}$ decreased when adding FAC, which reflected the formation of curcumin-Fe(III) complexes, and the lowest absorbance was observed when equal FAC was added (Figure 5). Moreover, cytotoxicity of curcumin was mostly neutralized by equal or greater amounts of FAC in DU145 and PC3 cells (Figure 6A and B, respectively), which provided additional evidence for the iron-chelating properties of curcumin. These results indicated that curcumin bound to FAC at a ratio of $\sim 1: 1$.

\section{FAC reversed curcumin-induced apoptosis and autophagy}

Under fluorescence microscopy, we found that characteristic changes in apoptosis induced by curcumin were mostly neutralized by equal amounts of FAC in DU145 and PC3 cells (Figure 2A and B, respectively). The results of FCM showed that the apoptosis rates of DU145 and

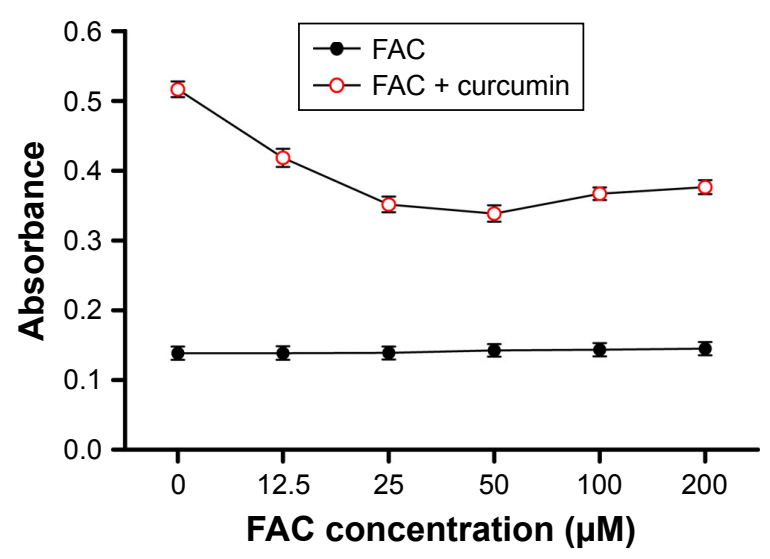

Figure 5 Curcumin binds to iron at a ratio of $\sim 1: 1$.

Notes: Increasing concentrations of FAC were incubated with or without curcumin $(50 \mu \mathrm{M})$. Then, the absorbance of the solution at $430 \mathrm{~nm}$ was detected. Results are expressed as mean $\pm S D$ of three independent experiments.

Abbreviation: FAC, ferric ammonium citrate.
PC3 were significantly reduced when pretreated with equal amounts of FAC (Figure 7A). Similarly, the evaluated expression levels of caspase-3, caspase-9 and LC3-II in DU145 and PC3 cells were mostly blocked by $50 \mu \mathrm{M}$ FAC (Figure 7B and C, respectively). FAC protected CRPC cells from curcumin-induced apoptosis and autophagy. Altogether, these results support the idea that apoptosis- and autophagy-inducing effects of curcumin are associated with its iron-chelating property.

\section{FAC reversed curcumin-induced increases in TfRI and IRPI activities}

To further confirm that apoptosis- and autophagy-inducing effects of curcumin are associated with its iron-chelating property, the intracellular status of iron was evaluated by the expression levels of TfR1, ferritin and IRP1. Under iron deprivation conditions, TfR1 is induced through IRP1mediated mRNA stabilization. ${ }^{4}$ As shown in Figure 8, levels of TfR 1 increased in response to $50 \mu \mathrm{M}$ curcumin treatment, which was consistent with IRP1 activation and reflected the reduction of intracellular iron levels in CRPC cells. Moreover, pretreatment with equal amounts of FAC blocked the iron deprivation induced by curcumin. These observations strongly suggest that an iron-chelating property contributed to apoptosis- and autophagy-inducing effects of curcumin.

\section{Discussion}

In this study, we have shown that curcumin demonstrated not only apoptosis- and autophagy-inducing activities but also efficient iron-chelating activity in CRPC cells. We speculated that curcumin causes iron deprivation by chelating iron. Iron deprivation, in turn, induces apoptosis and protective autophagy. Our studies suggest mechanistic information for the future development of curcumin to treat CRPC and provide a rationale on the use of iron deprivation for CRPC therapy.

Curcumin was previously reported to induce caspaserelated apoptosis in both androgen-dependent and castrationrefractory PCa cells, as opposed to autophagy in androgendependent PCa cells. ${ }^{4}$ Similarly, in our experimental approach, we found that curcumin induced caspase-related apoptosis in DU145 and PC3 cells in dose-dependent manners (Figures 1 and 2). We further demonstrated that exposure of CRPC cells to curcumin resulted in significant autophagy confirmed by both TEM and Western blots (Figure 3 ). Then, we used the autophagic inhibitor 3-MA and demonstrated that curcumin-induced autophagy protected CRPC cells from apoptosis (Figure 4). Similarly, induction of protective 
A

DU145

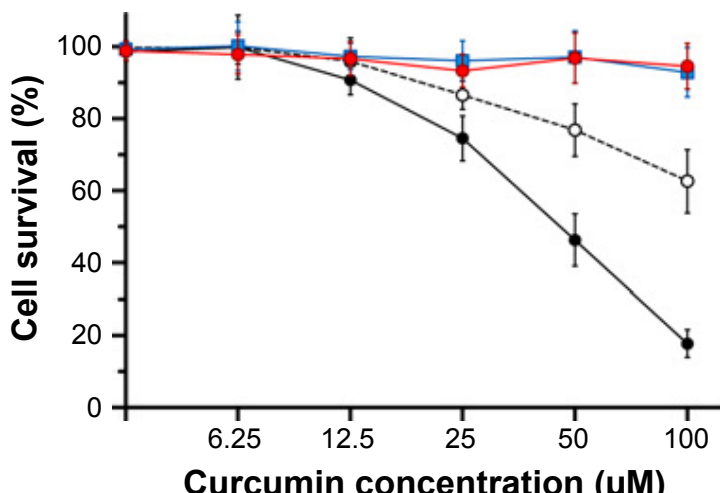

B

PC3

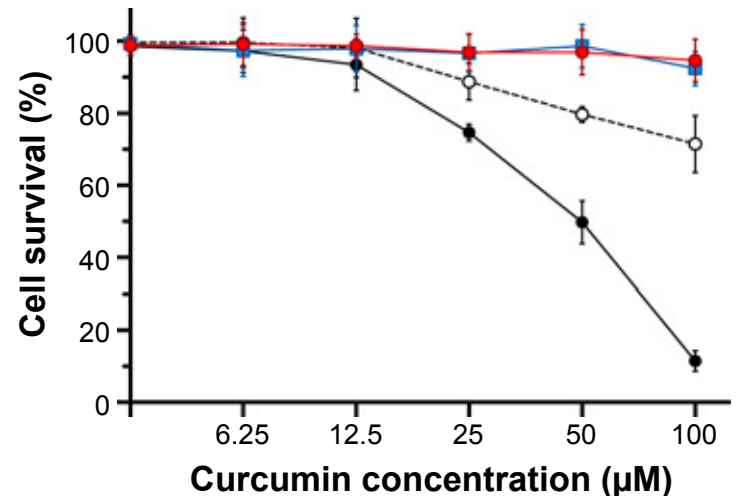

$\rightarrow$ Curcumin:FAC $=1: 0 \quad-0$ Curcumin:FAC $=3: 1 \rightarrow$ Curcumin:FAC $=1: 1 \rightarrow$ Curcumin:FAC $=1: 3$

Figure 6 Iron reduces the cytotoxicity of curcumin.

Notes: DUI45 (A) and PC3 (B) cells were incubated for $24 \mathrm{~h}$ with various concentrations of curcumin mixed with FAC at different ratios. The survival rate of DUI45 and PC3 cells was determined by MTT assay. Results are expressed as mean \pm SD of three independent experiments.

Abbreviations: FAC, ferric ammonium citrate; MTT, 3-(4,5-dimethylthiazol-2-yl)-2,5-diphenyltetrazolium bromide.

autophagy was observed in androgen-dependent PCa cells and glioblastoma cells after curcumin treatment. ${ }^{10,17,18}$

Obtaining a better understanding of the mechanisms that trigger apoptosis and autophagy is crucial for eradicating CRPC. Moustapha et al ${ }^{19}$ reported that curcumin induces crosstalk between autophagy and apoptosis mediated by calcium release from the endoplasmic reticulum (ER), lysosomal destabilization and mitochondrial events. Some studies have shown that curcumin is a biologically active iron chelator and induces cytotoxicity through iron chelation, ${ }^{10,17,18}$ indicating that curcumin may induce apoptosis and autophagy through its iron-chelating properties.
A
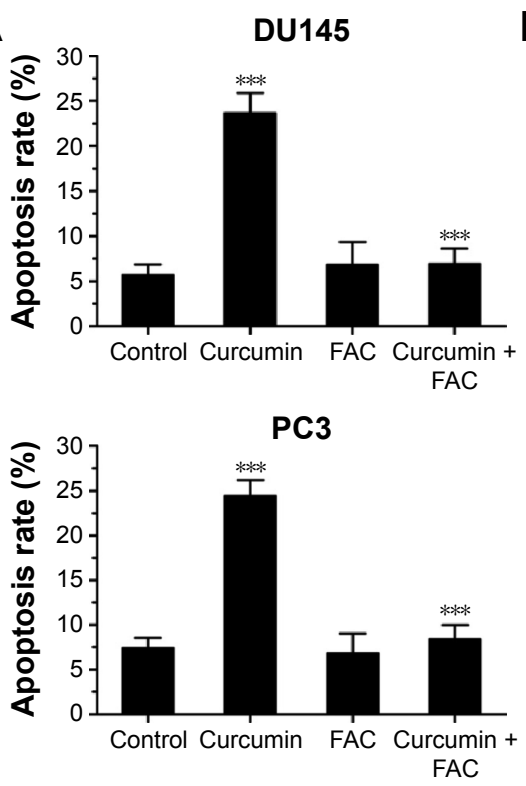

B

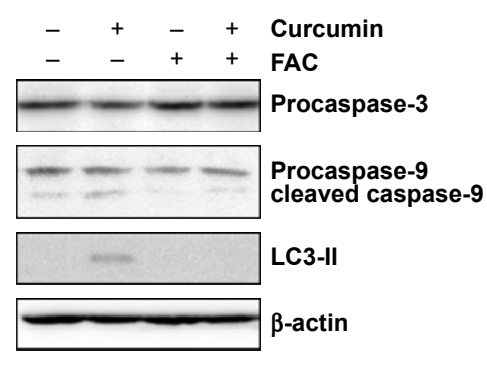

DU145

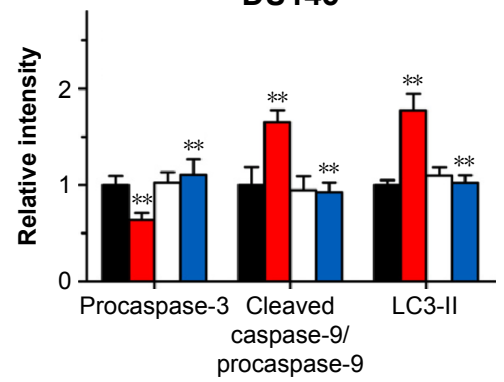

C
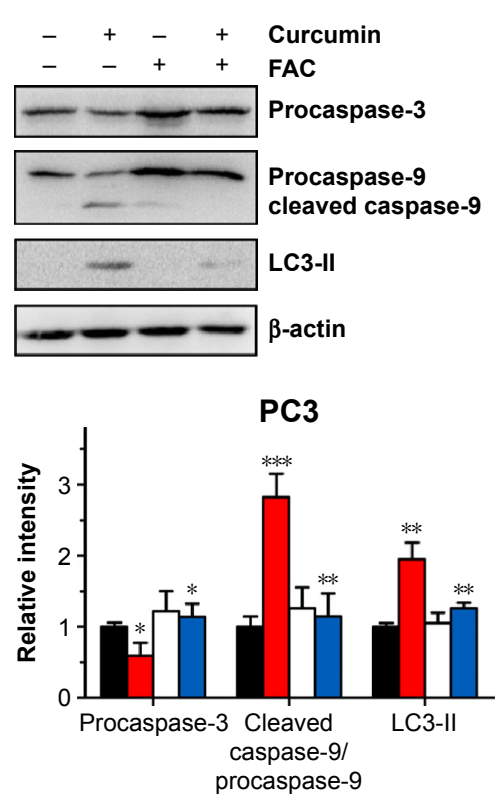

Control

Curcumin

Curcumin + FAC

Figure 7 FAC protects DUI45 and PC3 cells from curcumin-induced apoptosis and autophagy.

Notes: DUI45 and PC3 cells were incubated with curcumin $(50 \mu \mathrm{M})$ or combined treatment with FAC (50 $\mu$ M) for 24 h. (A) The percentage of apoptotic cells was analyzed by flow cytometric analysis of Annexin V and PI double staining. (B and C) Expression levels of caspase-3, caspase-9 and LC3-II were examined by Western blot analysis; (B) represent DUI45, (C) represent PC3. The data are expressed as mean \pm SD. $n=3, * P<0.05$, $* * P<0.0$ I and $* * * P<0.00$ I vs control or curcumin-treated group, by Student's $t$-test.

Abbreviations: FAC, ferric ammonium citrate; PI, propidium iodide; LC3-II, light chain 3-II. 
A

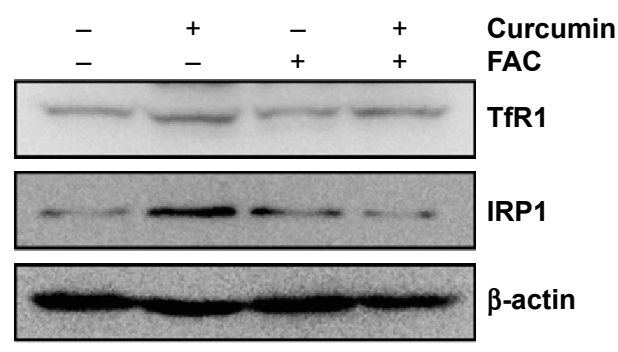

B

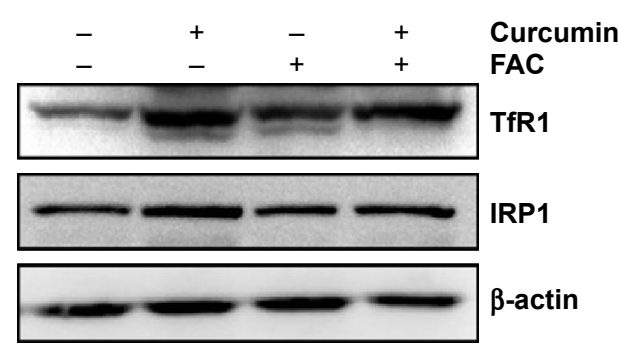

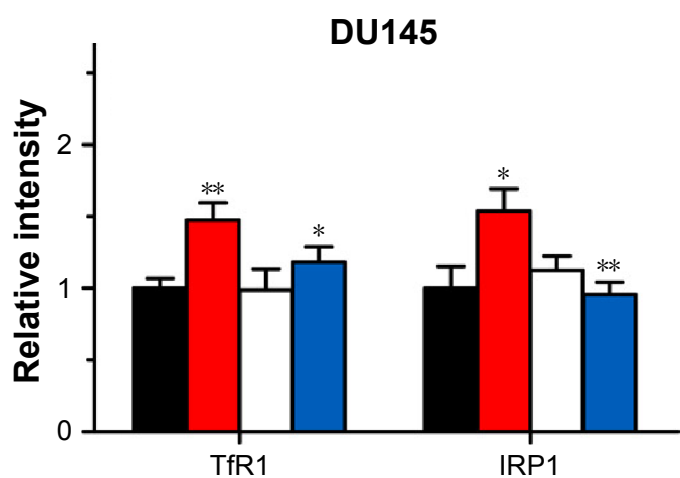

PC3

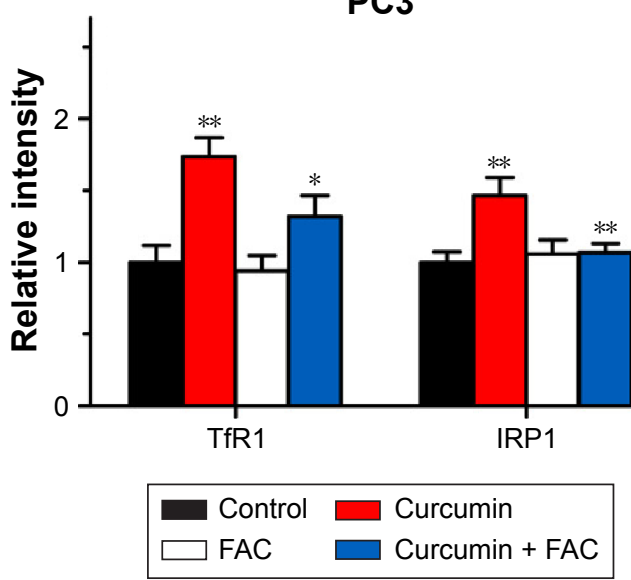

Figure 8 Iron deprivation induced by curcumin at apoptosis- and autophagy-inducing concentrations in DUI 45 and PC 3 cells.

Notes: DUI45 (A) and PC3 (B) cells were incubated with curcumin $(50 \mu \mathrm{M})$ or combined treatment with FAC (50 $\mu \mathrm{M})$ for 24 h, and expression levels of TfRI and IRPI were examined by Western blot analyses. $n=3, * P<0.05$ and $* * P<0.01$ vs control or curcumin-treated group, by Student's $t$-test.

Abbreviations: FAC, ferric ammonium citrate; TfRI, transferrin receptor I; IRPI, iron regulatory protein I.

Moreover, these authors have proposed the estimated structure of curcumin chelated with $\mathrm{FeCl}_{3}$ at a 1:1 molar ratio. Similarly, our experimental results indicated that curcumin chelates with FAC at a ratio of $\sim 1: 1$ in DMEM (Figures 5 and 6). In addition, we found that apoptosis and autophagy of DU145 and PC3 cells induced by curcumin were significantly inhibited with co-incubation of equal amounts of FAC (Figures 2 and 7). These results suggest that the apoptosis- and autophagy-inducing effects of curcumin are associated with its iron-chelating property. Furthermore, the expression of proteins participating in iron metabolism was examined. We found that curcumin-induced increases in TfR 1 and IRP1 activities were reversed by equal amounts of FAC, indicating that curcumin induced iron deprivation in CRPC cells by chelating iron (Figure 8 ). These observations strongly suggest that iron-chelating properties contributed to apoptosis- and autophagy-inducing effects of curcumin in CRPC cells. In fact, iron deprivation has been found to induce apoptosis and autophagy in a series of cell lines. ${ }^{20}$ However, we first confirmed that iron-chelating properties of curcumin contributed to its apoptosis- and autophagyinducing effects in tumor cells.

Autophagy is a cellular degradation mechanism to clear the cell of damaged or superfluous proteins and organelles, serving as an alternative material and energy source during periods of metabolic stress to maintain viability. Moreover, autophagy plays an important role in maintaining physiological iron balance in the cell, through its role in the degradation of the ironstorage protein ferritin. ${ }^{21}$ Similarly, previous studies showed that poorly permeant iron chelators led to lysosome-mediated degradation and iron extraction of ferritin via autophagy. ${ }^{22}$ In our experiment, curcumin caused iron deprivation by chelating iron. Iron deprivation, in turn, induced apoptosis and protective autophagy. These results indicate that autophagy induced by curcumin favors to maintain intracellular iron homeostasis and survival of CRPC cells.

\section{Conclusion}

We have shown that curcumin efficiently causes iron deprivation in DU145 and PC3 cells. Moreover, curcumin 
was highly effective for inducing apoptosis and protective autophagy in vitro. As expected, the role of iron deprivation in the apoptosis- and autophagy-inducing effects of curcumin was confirmed by iron rescue studies. Taken together, we demonstrated that curcumin induces apoptosis and protective autophagy in CRPC cells, which is at least partially dependent on its iron-chelating properties. Further experiments in vivo are needed to verify our findings.

\section{Acknowledgments}

The authors thank Assistant Professor Dr Bertram Yuh (City of Hope National Cancer Center, USA) and Associate Professor Dr Shu Zhang (Huazhong University of Science \& Technology, China) for helping revise the manuscript. This work was supported by the National Natural Science Foundation of China (NSFC; No 81101944 and No 81173608) and Natural Science Foundation of Hubei Province, China (No 2016CFB619).

\section{Disclosure}

The authors report no conflicts of interest in this work.

\section{References}

1. Torre LA, Bray F, Siegel RL, Ferlay J, Lortet-Tieulent J, Jemal A. Global cancer statistics, 2012. CA Cancer J Clin. 2015;65(2):87-108.

2. Heidenreich A, Bastian PJ, Bellmunt J, et al; European Association of Urology. EAU guidelines on prostate cancer. Part II: treatment of advanced, relapsing, and castration-resistant prostate cancer. Eur Urol. 2014;65(2):467-479.

3. Waldvogel-Abramowski S, Waeber G, Gassner C, et al. Physiology of iron metabolism. Transfus Med Hemother. 2014;41(3):213-221.

4. Kuhn LC. Iron regulatory proteins and their role in controlling iron metabolism. Metallomics. 2015;7(2):232-243.

5. Zhang C, Zhang F. Iron homeostasis and tumorigenesis: molecular mechanisms and therapeutic opportunities. Protein Cell. 2015;6(2): 88-100.

6. Ba Q, Hao M, Huang H, et al. Iron deprivation suppresses hepatocellular carcinoma growth in experimental studies. Clin Cancer Res. 2011; 17(24):7625-7633.

7. Pullarkat V, Meng Z, Donohue $C$, et al. Iron chelators induce autophagic cell death in multiple myeloma cells. Leuk Res. 2014;38(8):988-996.

8. Devassy JG, Nwachukwu ID, Jones PJ. Curcumin and cancer: barriers to obtaining a health claim. Nutr Rev. 2015;73(3):155-165.
9. Jordan BC, Mock CD, Thilagavathi R, Selvam C. Molecular mechanisms of curcumin and its semisynthetic analogues in prostate cancer prevention and treatment. Life Sci. 2016;152:135-144.

10. Teiten MH, Gaascht F, Cronauer M, Henry E, Dicato M, Diederich M. Anti-proliferative potential of curcumin in androgen-dependent prostate cancer cells occurs through modulation of the Wingless signaling pathway. Int J Oncol. 2011;38(3):603-611.

11. Bernabe-Pineda M, Ramirez-Silva MT, Romero-Romo MA, GonzalezVergara E, Rojas-Hernandez A. Spectrophotometric and electrochemical determination of the formation constants of the complexes Curcumin-Fe(III)-water and Curcumin-Fe(II)-water. Spectrochim Acta A Mol Biomol Spectrosc. 2004;60(5):1105-1113.

12. Jiao Y, Wilkinson JT, Di X, et al. Curcumin, a cancer chemopreventive and chemotherapeutic agent, is a biologically active iron chelator. Blood. 2009;113(2):462-469.

13. Qing Y, Shuo W, Zhihua W, et al. The in vitro antitumor effect and in vivo tumor-specificity distribution of human-mouse chimeric antibody against transferrin receptor. Cancer Immunol Immunother. 2006; 55(9):1111-1121.

14. Hou J, Wang D, Zhang R, Wang H. Experimental therapy of hepatoma with artemisinin and its derivatives: in vitro and in vivo activity, chemosensitization, and mechanisms of action. Clin Cancer Res. 2008;14(17): 5519-5530.

15. van Heerde WL, Robert-Offerman S, Dumont E, et al. Markers of apoptosis in cardiovascular tissues: focus on Annexin V. Cardiovasc Res. 2000;45(3):549-559.

16. Mathur A, Abd EZ, Liu X, et al. Subverting ER-stress towards apoptosis by nelfinavir and curcumin coexposure augments docetaxel efficacy in castration resistant prostate cancer cells. PLoS One. 2014;9(8): e103109.

17. Cheng $Y$, Ren $X, Z$ Zhang $Y$, et al. Integrated regulation of autophagy and apoptosis by EEF2K controls cellular fate and modulates the efficacy of curcumin and Velcade against tumor cells. Autophagy. 2013;9(2): 208-219.

18. Zanotto-Filho A, Braganhol E, Klafke K, et al. Autophagy inhibition improves the efficacy of curcumin/temozolomide combination therapy in glioblastomas. Cancer Lett. 2015;358(2):220-231.

19. Moustapha A, Pérétout PA, Rainey NE, et al. Curcumin induces crosstalk between autophagy and apoptosis mediated by calcium release from the endoplasmic reticulum, lysosomal destabilization and mitochondrial events. Cell Death Discov. 2015;26(1):15017.

20. Ullio C, Brunk UT, Urani C, et al. Autophagy of metallothioneins prevents TNF-induced oxidative stress and toxicity in hepatoma cells. Autophagy. 2015;11(12):2184-2198.

21. Kurz T, Gustafsson B, Brunk UT. Cell sensitivity to oxidative stress is influenced by ferritin autophagy. Free Radic Biol Med. 2011;50(11): $1647-1658$.

22. Asano T, Komatsu M, Yamaguchi-Iwai Y, Ishikawa F, Mizushima N, Iwai K. Distinct mechanisms of ferritin delivery to lysosomes in iron-depleted and iron-replete cells. Mol Cell Biol. 2011;31(10): 2040-2052.

\section{Publish your work in this journal}

Drug Design, Development and Therapy is an international, peerreviewed open-access journal that spans the spectrum of drug design and development through to clinical applications. Clinical outcomes, patient safety, and programs for the development and effective, safe, and sustained use of medicines are the features of the journal, which

\section{Dovepress}

has also been accepted for indexing on PubMed Central. The manuscript management system is completely online and includes a very quick and fair peer-review system, which is all easy to use. Visit http://www.dovepress.com/testimonials.php to read real quotes from published authors. 